\title{
A forkhead gene, FoxE3, is essential for lens epithelial proliferation and closure of the lens vesicle
}

\author{
Åsa Blixt, ${ }^{1}$ Margit Mahlapuu, ${ }^{1}$ Marjo Aitola, ${ }^{2}$ Markku Pelto-Huikko, ${ }^{2}$ Sven Enerbäck, ${ }^{1}$ \\ and Peter Carlsson ${ }^{1,3}$ \\ ${ }^{1}$ Department of Molecular Biology, Göteborg University, The Lundberg Laboratory, Medicinaregatan 9C, Box 462, S-405 30 \\ Göteborg, Sweden; ${ }^{2}$ Department of Developmental Biology, Medical School, University of Tampere, Tampere, Finland
}

In the mouse mutant dysgenetic lens (dyl) the lens vesicle fails to separate from the ectoderm, causing a fusion between the lens and the cornea. Lack of a proliferating anterior lens epithelium leads to absence of secondary lens fibers and a dysplastic, cataractic lens. We report the cloning of a gene, FoxE3, encoding a forkhead/winged helix transcription factor, which is expressed in the developing lens from the start of lens placode induction and becomes restricted to the anterior proliferating cells when lens fiber differentiation begins. We show that FoxE3 is colocalized with $d y l$ in the mouse genome, that $d y l$ mice have mutations in the part of FoxE3 encoding the DNA-binding domain, and that these mutations cosegregate with the dyl phenotype. During embryonic development, the primordial lens epithelium is formed in an apparently normal way in dyl mutants. However, instead of the proliferation characteristic of a normal lens epithelium, the posterior of these cells fail to divide and show signs of premature differentiation, whereas the most anterior cells are eliminated by apoptosis. This implies that FoxE3 is essential for closure of the lens vesicle and is a factor that promotes survival and proliferation, while preventing differentiation, in the lens epithelium.

[Key Words: forkhead; lens epithelium; dysgenetic lens; FoxE3; cataract]

Received October 5, 1999; revised version accepted November 26, 1999.

Lens formation during vertebrate eye development is a classic example of induction. (Spemann 1901) The optic vesicle-an outgrowth from the ventral forebrain that later forms the retina-induces the surface ectoderm to invaginate and bud off the lens vesicle. A polarity soon develops in the primitive lens; posterior cells differentiate into elongated, crystallin-expressing, primary lens fibers that gradually fill the vesicle, whereas the anterior surface retains a layer of undifferentiated, proliferating cells. These cells of the anterior lens epithelium continue to divide throughout life and as they migrate posteriorly into the equatorial region of the lens, they start to differentiate into secondary lens fibers (McAvoy 1980; Piatigorsky 1981).

Several transcription factors are essential for lens development. The homeodomain proteins $\mathrm{Lhx} 2, \mathrm{Rx}$, and Six3 are involved in formation of and induction by the optic vesicle (Oliver et al. 1995; Mathers et al. 1997; Porter et al. 1997; Loosli et al. 1999). Lens-forming competence of the head ectoderm depends on the paired homeodomain protein Pax6 (Fujiwara et al. 1994; Gehring 1996; Furuta and Hogan 1998) and heterozygous loss of function of $P A X 6$ is responsible for aniridia in humans (Jordan et al. 1992; Glaser et al. 1994). Necessary com-

${ }^{3}$ Corresponding author.

E-MAIL peter.carlsson@molbio.gu.se; FAX +46 317733801. ponents of lens fiber differentiation and maturation include growth arrest and crystallin accumulation. The basic leucine zipper (bZIP) protein L-Maf (Ogino and Yasuda 1998) and Sox high mobility group (HMG) domain proteins (Kamachi et al. 1998; Nishiguchi et al. 1998) activate $\alpha$-and $\gamma$-crystallin gene expression, respectively. Cell cycle arrest requires the retinoblastoma protein (Morgenbesser et al. 1994) and the cyclin-dependent kinase $(C d k)$ inhibitors $C d k n 1 b\left(p 27^{\text {Kip } 1}\right)$ and $C d k n 1 c$ $\left(p 57^{\text {Kip2 }}\right)$ (Zhang et al. 1998), which depend on the homeodomain protein Proxl for their expression (Wigle et al. 1999). The cues that induce lens polarity, epithelial proliferation, and fiber differentiation are differences in growth factor content between the aqueous and vitreous humor (Hyatt and Beebe 1993; Schulz et al. 1993; Klok et al. 1998; Potts et al. 1998).

Here we report the cloning of a forkhead transcription factor gene, FoxE3, which is expressed in the lens epithelium. We show that FoxE3 is mutated in a classic mouse model with defect lens development.

Results

Cloning of FoxE3

In a screen for forkhead genes from a human genomic library (Pierrou et al. 1994), previously we isolated a par- 
Blixt et al.

tial clone containing a novel forkhead motif that we named FREAC8 (FOXE3 according to the latest nomenclature) (Larsson et al. 1995). To locate the spatial and temporal distribution of expression, we isolated genomic clones of the mouse homolog to perform in situ hybridization. Following mouse gene nomenclature recommendations, we call this gene FoxE3. Sequencing of $7 \mathrm{~kb}$ around the forkhead motif of FoxE3 revealed a single open reading frame of 864 nucleotides, corresponding to a 288-amino-acid protein (Fig. 1). Comparison with the sequence of human FOXE3 cDNA clones (A Blixt, M. Ormestad, M. Sparacio, and P. Carlsson, unpubl.) confirmed that FoxE3 lacks introns and verified the predicted borders of the coding sequence. The amino acid sequence of FoxE3 has no obvious similarity to other proteins in the databases, outside the forkhead domain.
FoxE3 is expressed in the lens

Whole-mount in situ hybridization of mouse embryos from E8.5 to E10.75 and sections of embryos and adult tissues showed that FoxE3 is expressed in the lens of the developing eye (Fig. 2). Expression is first detected as a small dot on the surface ectoderm around E9.5 (Fig. 2a) and then rapidly increases as the lens placode is formed (Fig. 2b,g). FoxE3 expression becomes confined to the lens vesicle as this structure detaches from the surface ectoderm and initially it is evenly distributed throughout the vesicle (Fig. 2f). In situ hybridization of sections through the developing lens showed that FoxE3 expression is switched off in posterior cells of the lens vesicle as these cells start to differentiate to lens fibers, but remains high in the anterior cells (Fig. 2h). This pattern of

$\mathbf{A}$

\begin{tabular}{rllll|}
1 & MDAQVAFSGF & PALPSLTPSG & PQLPTLAGAE & PGREPEEVVG \\
41 & GGDAEPTAVP & GPGKRRRRPL & QRGKPPYSYI & ALIAMALAHA \\
81 & PGRRLTLAAI & YRFITERFAF & YRDSPRKWQN & SIRHNLTLND \\
121 & CFVKVPREPG & NPGKGNYWTL & DPAAADMFDN & GSFLRRRKRF \\
161 & KRAELPAPPP & PPPPFPYAPF & PPPPAPASAP & PARLFRLDSL \\
201 & LGLQPEPPGP & VAPEPPCCAA & PDAAFPPCAA & AASPPLYSPA \\
241 & SERLGLPAPL & PAQPLLALAG & SAGALGPLGA & GEAYLRQPGF \\
281 & APGLERYL & & &
\end{tabular}

B

Fox:3

FOxD1

FoxD4

FOxD3

FoxB2

FOxC2

FoxC1

FoxB1

FOXA3

FoxI1

FoxL1

FoxA1

FOxA2

FoxF 1

FoxG1

FoxJ1

Foxk1

FOX: 3

FOXD1

FoxD4

FoxD3

FoxB2

FoxC2

FoxC1

FoxB1

FOXA3

FoxI1

FoxL1

FoxA1

FOXA2

FoxF 1

FoxG1

FoxJ1

Foxk1

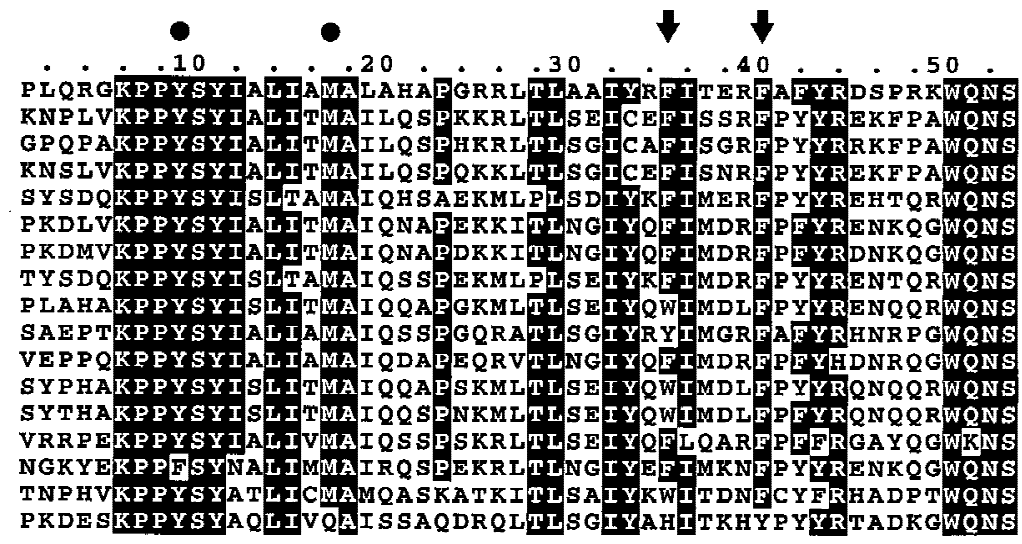
PKDE SKPPY SYAQLIVQASIS SAQDRQLTLSGIYAHITKHY PYYRTADKGWONS

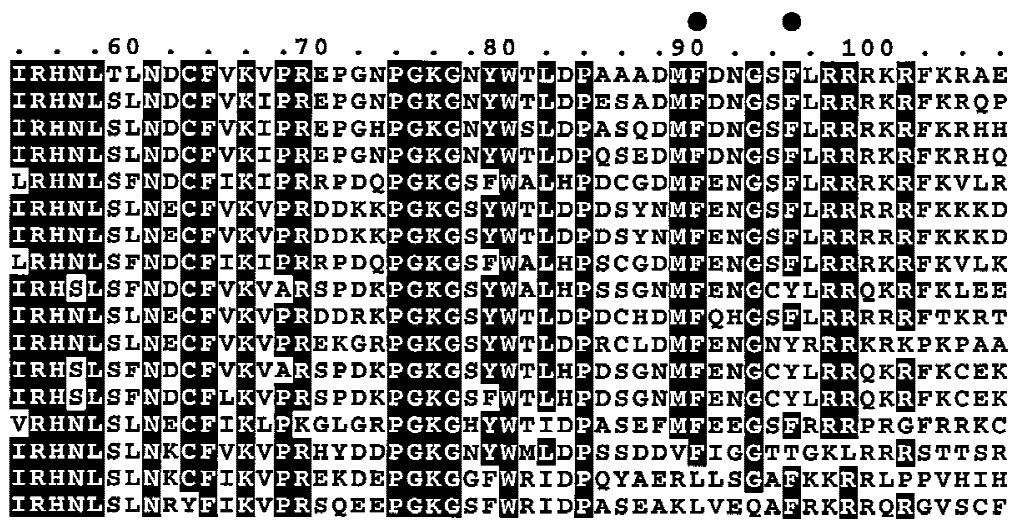

Figure 1. (a) Amino acid sequence of FoxE3 predicted from DNA sequence. The forkhead domain is boxed. $(b)$ The forkhead domain of FoxE3 aligned with other mouse forkhead proteins in the SwissProt database. Phe93 and Phe98 of FoxE3 are indicated with arrows and other amino acids in the methionine-aromatic rosette with solid circles. For conversion between the Fox nomenclature and old names, see http://www.biology.pomona.edu/fox.html. 

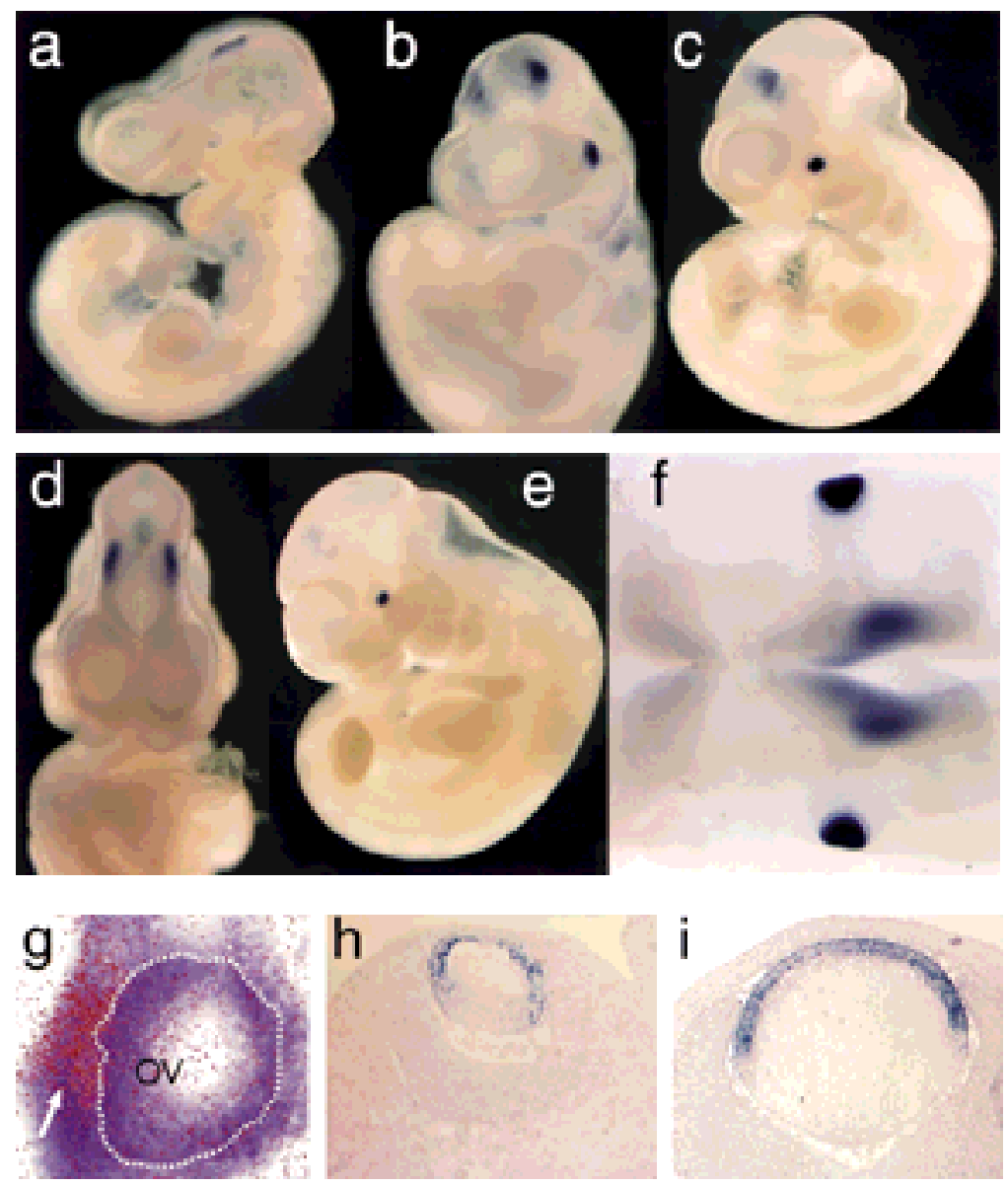

Figure 2. FoxE3 expression in embryonic development. (a) Whole mount in situ hybridization of 23somite mouse embryo showing the first signs of FoxE3 expression on the lateral head ectoderm and on the cephalic neural folds. (b) 25-somite stage embryo (E9.5) with expression in lens placode and caudal forebrain. $(c)$ Side view and $(d)$ frontal view of 30-somite embryo (E10.25) with high expression in lens vesicle and weaker expression in caudal, dorsolateral diencephalon. (e) 35-somite embryo (E10.5) with high expression in lens vesicle and fading brain expression. $(f)$ Dorsal view of clarified 30-somite embryo showing FoxE3 expression in lens vesicles and diencephalon (out of focus). ( $g$ ) Radioactive in situ hybridization showing lens placode (arrow) expression of FoxE3 (red) in section of E9.5 embryo. Dotted line indicates the border of the optic vesicle (OV). (h) Section through the eye of E12.5 embryo showing FoxE3 expression in the anterior part of the lens vesicle, but turned off in the differentiating primary lens fibers in the posterior part. (i) Section through the eye of E14.5 embryo demonstrating FoxE3 expression confined to the anterior epithelium of the lens.
FoxE3 expression, limited to the undifferentiated cells covering the anterior surface of the lens (Fig. 2i), is retained throughout embryogenesis and into adulthood. Apart from the lens, FoxE3 expression is seen in only one location during a brief period of development. It first appears on the neural folds in the cephalic region at about the same time as expression in the lateral head ectoderm (Fig. 2a). After closure of the anterior neuropore, expression is found in the most caudal, dorsolateral parts of the diencephalon (Fig. 2b,c,d). The expression level peaks at the 25- to 30-somite stage (E9.5-E10) and then rapidly vanishes. From E11.5, in situ hybridization failed to detect FoxE3 expression at any developmental stage in any tissue, except for in the anterior lens epithelium.

\section{FoxE3 is colocalized with dysgenetic lens}

Given its early onset and restricted expression pattern, combined with the well-documented role of forkhead genes in embryogenesis (for review, see Kaufmann and Knöchel 1996), FoxE3 was an obvious candidate gene for developmental lens defects. Previously, we have localized the human homolog FOXE3 to 1p32 (Larsson et al. 1995) and although no human lens disorder has been linked to this chromosomal region, the mouse mutation dysgenetic lens (dyl) (Sanyal and Hawkins 1979) has been mapped to an area of chromosome 4 syntenic with human 1p32 (Sanyal et al. 1986; Jänne et al. 1995). dyl arose spontaneously in Balb/c mice and segregates as an autosomal recessive trait. The most striking phenotype in this mutant is a persistent connection between the lens and the corneal epithelium (Fig. 3f), which results from failure of the lens vesicle to close and separate from the ectoderm (Fig. 3b; Sanyal and Hawkins 1979). Reduced size, irregular shape, and disorganized structure with large vacuoles are other characteristics of the lenses of $d y l$ mice (Fig. 3; Sanyal and Hawkins 1979). Lens fiber elongation and expression of crystallins are initiated in an apparently normal way (Brahma and Sanyal 1984, 1987), but the number of fibers is drastically reduced, resulting in a diminutive, irregular, and cataractic lens where lens fiber material is sometimes expelled to the exterior through the persistent ectodermal connection (Fig. 3b). In wild-type lens, a well-defined epithelial layer is seen on the anterior surface (Fig. 3e), which is replaced by elongated cells posterior of the equatorial zone (Fig. $3 \mathrm{~g})$. In $d y 1$ lens, the epithelial layer is formed during lens vesicle polarization, but then gradually disappears and is absent by the time of birth, leaving a cataractic lens where cell morphology is similar on either side of the lens equator (Fig. 3h). Apart from the eye defects, dyl mice appear to be normal. 

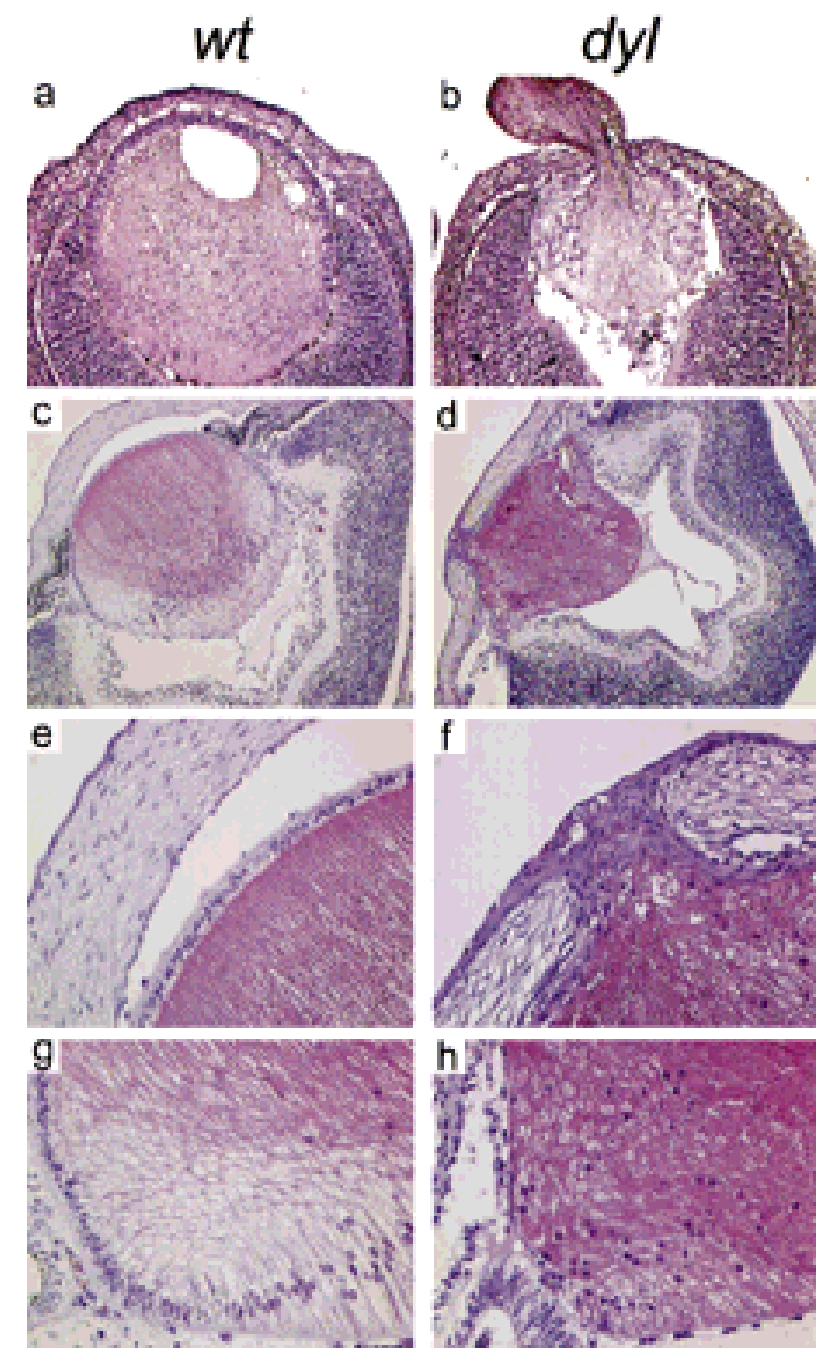

Figure 3. Hematoxylin and eosin-stained sections through the eye of wild-type $(a, c, e, g)$ and $d y l(b, d, f, h)$ mice. $(a, b)$ Eyes from E14.5 embryos. The lens vesicle fails to close in $d y 1$ mutants, which in some cases leads to lens material being ejected to the exterior $(b) .(c-h)$ Eyes from newborn mice. Note the small, irregularly shaped lens $(d)$ and the persistent connection between lens and cornea $(d, f)$ in $d y l$ mutants. In the equatorial zone of the lens from wild-type animals $(g)$ there is a transition from the well-developed anterior epithelium to posterior elongated lens fibers. In $d y l$ mutants $(h)$ no clear difference is seen between anterior and posterior side and a defined epithelium is missing.

To establish whether FoxE3 is located in the chromosomal region to which $d y l$ has been mapped, we localized FoxE3 with fluorescent in situ hybridization (FISH). As shown in Figure 4, the cytogenetic position of FoxE3 is $4 \mathrm{C7}$, which is in perfect agreement with the genetic mapping of $d y l$ to linkage position $49.6 \mathrm{cM}$ of chromosome 4 (Sanyal et al. 1986; Jänne et al. 1995).

\section{dyl mutants have mutations in FoxE3}

The entire coding region plus $600 \mathrm{bp}$ of flanking sequences of FoxE3 were determined from a homozygous dyl mouse. Comparison with the previously determined wild-type sequence revealed five single nucleotide substitutions, three of which alter the predicted amino acid sequence of FoxE3. One missense mutation substitutes a glutamic acid for glutamine at position 253 in the carboxy-terminal part of the protein and the other two occur close to each other within the forkhead box. Both the latter are $\mathrm{T} \rightarrow \mathrm{C}$ transitions that result in phenylalanines being replaced by other amino acids: Phe-93-Leu and Phe-98-Ser (Fig. 4c). The $d y l$ mutant is maintained on a $\mathrm{Balb} / \mathrm{c}$ genetic background, whereas our wild-type sequence was derived from 129/Sv DNA. To determine whether the observed mutations are restricted to the $d y 1$ mutant, or reflects differences between the two inbred strains, we also sequenced FoxE3 from a normal Balb/c mouse. The two translationally silent mutations and the Gln-253-Glu substitution turned out to be differences between Balb/c and 129/Sv, whereas the two mutations in the forkhead box of FoxE3 are specific for the $d y 1$ mutant.

\section{The dyl phenotype cosegregates with FoxE3 mutations}

The progeny from sibling matings of $d y l /+$ mice, obtained from $d y l / d y l \times$ Balb $/ c$ crossings, were scored for the $d y l$ phenotype by visual inspection of the lens under stereomicroscope ( 2 weeks postpartum or later) or by microscopic examination of histological sections (embryos or newborn pups). Each individual was also genotyped with regard to the $\mathrm{T} 277 \mathrm{C}$ mutation /corresponding to the Phe-93-Leu substitution) in the forkhead box of FoxE3. As shown in Table 1, only FoxE3 ${ }^{T 277 C} /$ FoxE3 $3^{T 277 C}$ homozygotes exhibited the lens defects characteristic of dyl mutants.

\section{The lens epithelium fails to proliferate in dyl mutants}

In situ hybridization of sections through developing eyes from $d y 1$ embryos showed that FoxE3 is expressed in an apparently normal way in this mutant during early lens development. At later stages the morphological differences between wild-type and mutant lens make a comparison difficult; hardly anything remains of the lens epithelium in $d y 1$ embryos at time points later than E15.5. At E14.5, however, the epithelium has become well defined and the general morphology of $d y 1$ and wild-type lenses remain similar enough to allow a direct comparison. At this stage FoxE3 expression is confined to the anterior lens epithelium of both wild-type and mutant (Fig. 5a,b).

The lens morphology of $d y l$ mice suggests that the primary defect, leading to a drastic reduction in the number of secondary lens fibers, is failure of the lens epithelium to proliferate. To investigate this, we assayed DNA synthesis in the developing eye of E14.5 and E15.5 embryos by BrdU incorporation. In the anterior epithelium of wild-type lenses from both time points, a high percentage of the cells had gone through S-phase and stained BrdU positive (Fig. 5c,e). In lenses from E14.5 dyl em- 
A

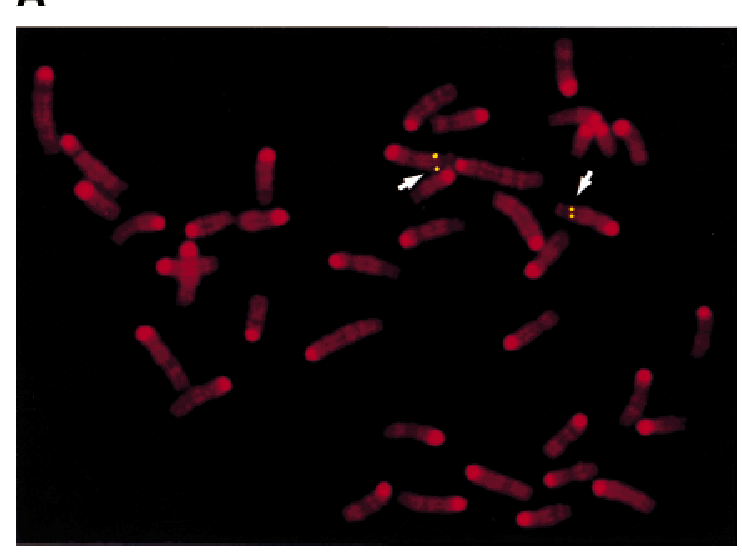

C

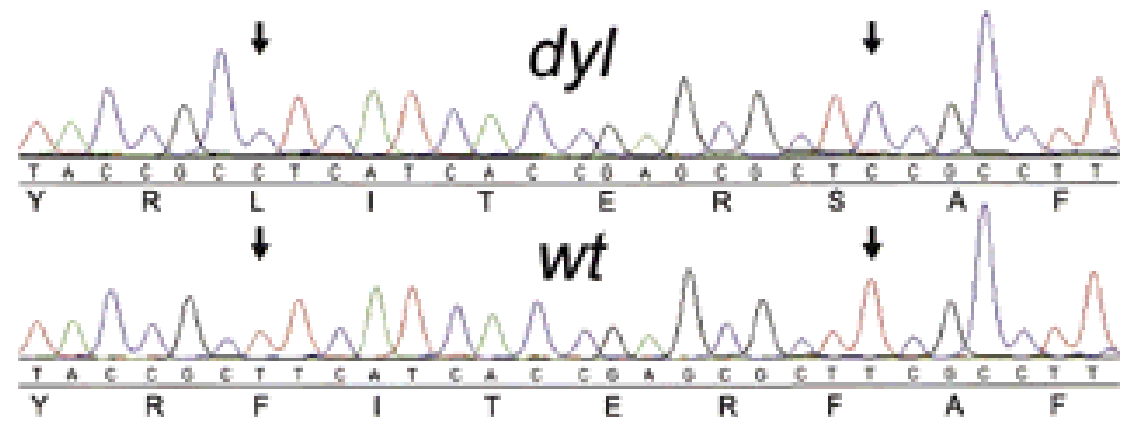

B

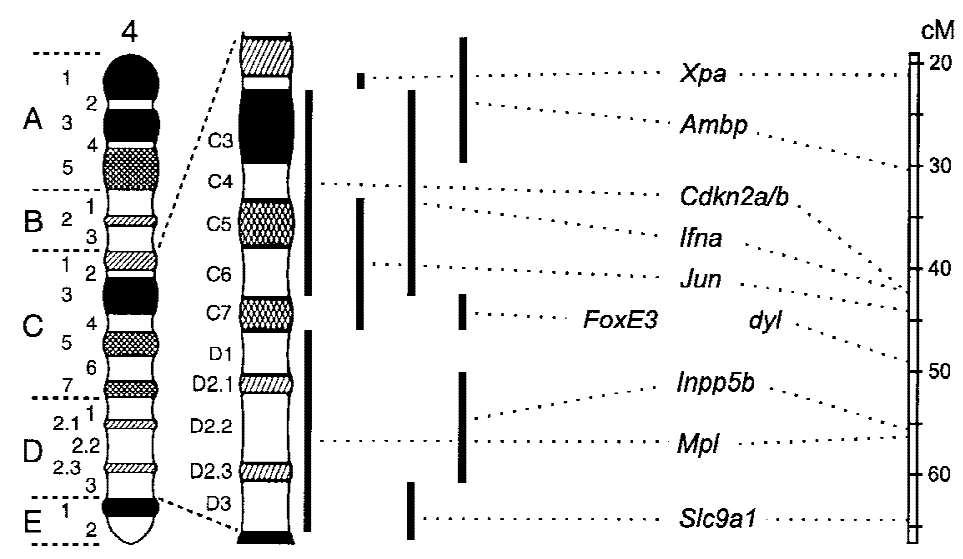

Figure 4. FoxE3 and $d y l$ are colocalized in the mouse genome. (a) Localization of FoxE3 to 4C7 on mouse metaphase chromosomes by fluorescence in situ hybridization (FISH). (b) Comparison between the cytogenetic and genetic maps of mouse chromosome 4. Genes that cytogenetically map proximally or distally of FoxE3 have the same location relative to $d y l$ on the genetic map. Map data, except FoxE3, are taken from the Mouse Genome Database (http:// www.informatics.jax.org/l. (c) DNA sequence from the forkhead box of FoxE3. dyl mice have two missense mutations that substitute leucine and serine for Phe-93 and Phe-98, respectively. bryos a morphologically distinguishable epithelium is present (for example, see Fig. 5b), but only a few cells had incorporated BrdU (Fig. 5d). At E15.5 no BrdU-positive cells could be detected in the lens (Fig. 5f). Consistent with this, the expression of proliferation markers also differ between $d y l$ and wild-type. The lens epithelium from wild-type embryos contains mRNA for Mki67 (Fig. $5 \mathrm{~g}$ ) and PCna (Fig. 5i,k), encoding proliferation associated antigens, with the highest expression levels found in the germinative zone next to the neural retina. In $d y 1 \mathrm{mu}-$ tants, expression of these genes is barely detectable and the residual expression has a more anterior distribution (Fig. 5h,j,1).

\section{dyl lens epithelial cells are eliminated}

through premature differentiation and apoptosis

In a normal developing lens, a distinct border separates the dividing cells of the epithelium from the posterior, differentiating cells and this border coincides with the limit for FoxE3 expression. A possible role for FoxE3, compatible with the lack of epithelial growth in the $d y 1$ mutant, could be to maintain this border by preventing the expression in epithelial cells of genes involved in growth inhibition and differentiation. Cdkn1c ( $\left.=p 57^{K I P 2}\right)$ encodes an inhibitor of Cdks and is highly expressed in the equatorial zone of the lens, where cell cycle exit occurs (Fig. 6a; Zhang et al. 1998). In wild-type E14.5 lens there is no overlap in expression between FoxE3 and Cdkn1c (Figs. 5a and 6a,c), whereas in the $d y 1$ mutant the expression of $C d k n 1 c$ extends anteriorly into the epithelium (Fig. 6b,d). In addition, the expression level of $C d k n 1 c$ is much lower in $d y l$ lens, approximately equal to that near the tip of the neural retina (Fig. $6 \mathrm{~b}, \mathrm{~d})$, whereas the wild-type expression is comparable to that in the periocular chondrogenic mesenchyme (Fig. 6a,c). The homeodomain protein Prox1 is essential for $C d k n 1 c$ expression in lens, as shown by the absence of Cdkn1c mRNA in lenses of Prox1 null mice (Wigle et al. 1999). Prox1 is expressed in the entire lens, but only

Table 1. Cosegregation of the FoxE $3^{\mathrm{T} 277 \mathrm{C}}$ mutation and the dyl phenotype

\begin{tabular}{lccc}
\hline & $+/+$ & FoxE3 & FoxE3 \\
\hline Connection between lens and cornea. Small cataractic lens. & 0 & 0 & 24 \\
No connection between lens and cornea. Lens not cataractic. & 23 & 52 & 0 \\
\hline
\end{tabular}

Progeny from $d y 1 /+\times d y l /+$ crossings were scored for the $d y l$ phenotype and were genotyped with regard to the FoxE $3^{T 277 C}$ mutation. The number of individuals in each category is indicated. 


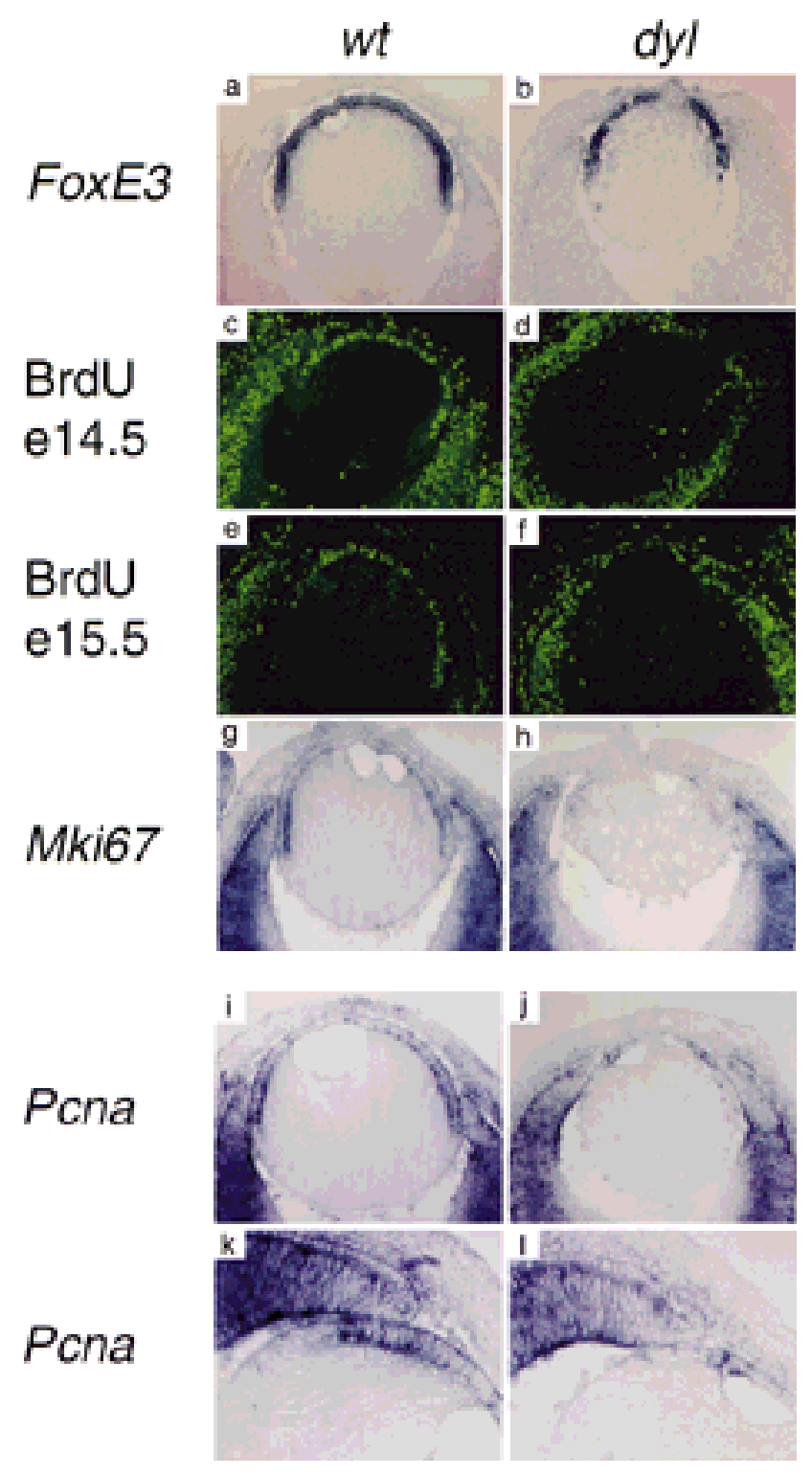

Figure 5. The lens epithelium fails to proliferate in $d y \mathrm{l} \mathrm{mu-}$ tants. In situ hybridization show similar patterns of FoxE3 expression in wild-type $(a)$ and $d y l(b)$ E14.5 lens. BrdU incorporation reveals intense proliferation of epithelial cells in wildtype lens $(c, e)$, but a sparse distribution of replicating cells in E14.5 dyl lens $(d)$. At E15.5 the difference is even more pronounced with only a few BrdU-positive cells in the mutant $(f)$. The expression of genes encoding proliferation associated antigens Mki67 $(g, h)$ and Pcna (i-l) is down-regulated and displaced anteriorly in $d y l$ mutants. All sections except $e$ and $f$ are from stage E14.5.

weakly in the epithelium and with the highest mRNA levels found in the equatorial zone, coinciding with the site of Cdkn1c expression (Fig. 6e). In the dyl mutant lens, the high level of Prox1 expression extends anteriorly into the epithelium and, like $C d k n 1 c$, overlaps with the expression of FOxE3 (Fig. 6f).

Differentiation is closely linked to cell cycle arrest in lens development. The Crya1 gene, encoding $\alpha 1$-crystal- lin, is expressed at a low level in the epithelial cells, but is induced transcriptionally to very high levels in the equatorial zone and stays high in the differentiating lens fibers (Fig. $6 \mathrm{~g}$ ). In the $d y 1$ mutant, epithelial cells express Crya1 at about the same level as the rest of the lens and no induction is seen in the equatorial zone (Fig. 6h). Only in the most anterior epithelial cells is the Crya1 expression lower. E-cadherin is expressed throughout the lens epithelium and this expression is retained until the cells reach the equatorial zone and start to differentiate (Fig. 6i). The presence of E-cadherin-expressing cells on the anterior surface of the lens from E14.5 dyl mice verify the presence at this stage of an epithelial layer, despite the lack of proliferation (Fig. 6j). In the mutant, expression of E-cadherin does not extend as far posteriorly as in the wild-type lens, which, together with the altered expression pattern of Crya1, show that the border between differentiated and undifferentiated cells is moved forward.

Platelet-derived growth factor (PDGF) A is secreted by the iris and the ciliary body, next to the germinative zone of the lens (Orr-Urtreger and Lonai 1992; Reneker and Overbeek 1996) and the Pdgfra gene, encoding the PDGF $\alpha$ receptor, is expressed in the lens epithelium (Fig. 6k,m; Morrison-Graham et al. 1992; Orr-Urtreger and Lonai 1992). In the dyl mutant, Pdgfra expression is diminished compared to wild-type and the residual expression is restricted to the most anterior part of the epithelium (Fig. 61,n). The lack of proliferation and the downregulation of Pdgfra expression suggest that lens epithelial cells in the $d y l$ mutant may be experiencing a decreased growth factor receptor signaling. Because many cell types are protected from apoptosis by growth factor stimulation, we investigated whether the curtailed growth of the lens epithelium in $d y l$ mutants was accompanied by programmed cell death. No signs of apoptosis could be detected in wild-type E14.5 lens, whereas the $d y l$ mutant lens had many apoptotoic cells in the anterior part of the epithelium (Fig. 6o,p).

\section{Discussion}

Of 70 forkhead proteins in the SwissProt database described from metazoans and Saccharomyces, 67 have an aromatic amino acid in the position that corresponds to Phe-93 in FoxE3. The other site of mutation in $d y l$ mice, Phe-98, is even more conserved. Here, 62 of 70 proteins have phenylalanine and 5 additional proteins have other aromatic amino acids. In the cocrystal structure of the forkhead protein FoxA3 (HNF3 $\gamma$ ) bound to DNA (Clark et al. 1993), the aromatic residues corresponding to Phe93 and Phe-98 of FoxE3 are located in helix 2 of the forkhead helix-turn-helix motif and in the $\mathrm{T}^{\prime}$ loop connecting helix 2 and helix 3 . Together with conserved aromatic residues from the amino-terminal end of the forkhead domain and from the carboxy-terminal second wing (W2), these amino acids generate a rosette-like structure in which a conserved methionine is surrounded by five aromatic side chains (Fig. 1b; Clark et al. 1993). A few proteins distantly related to the forkhead 


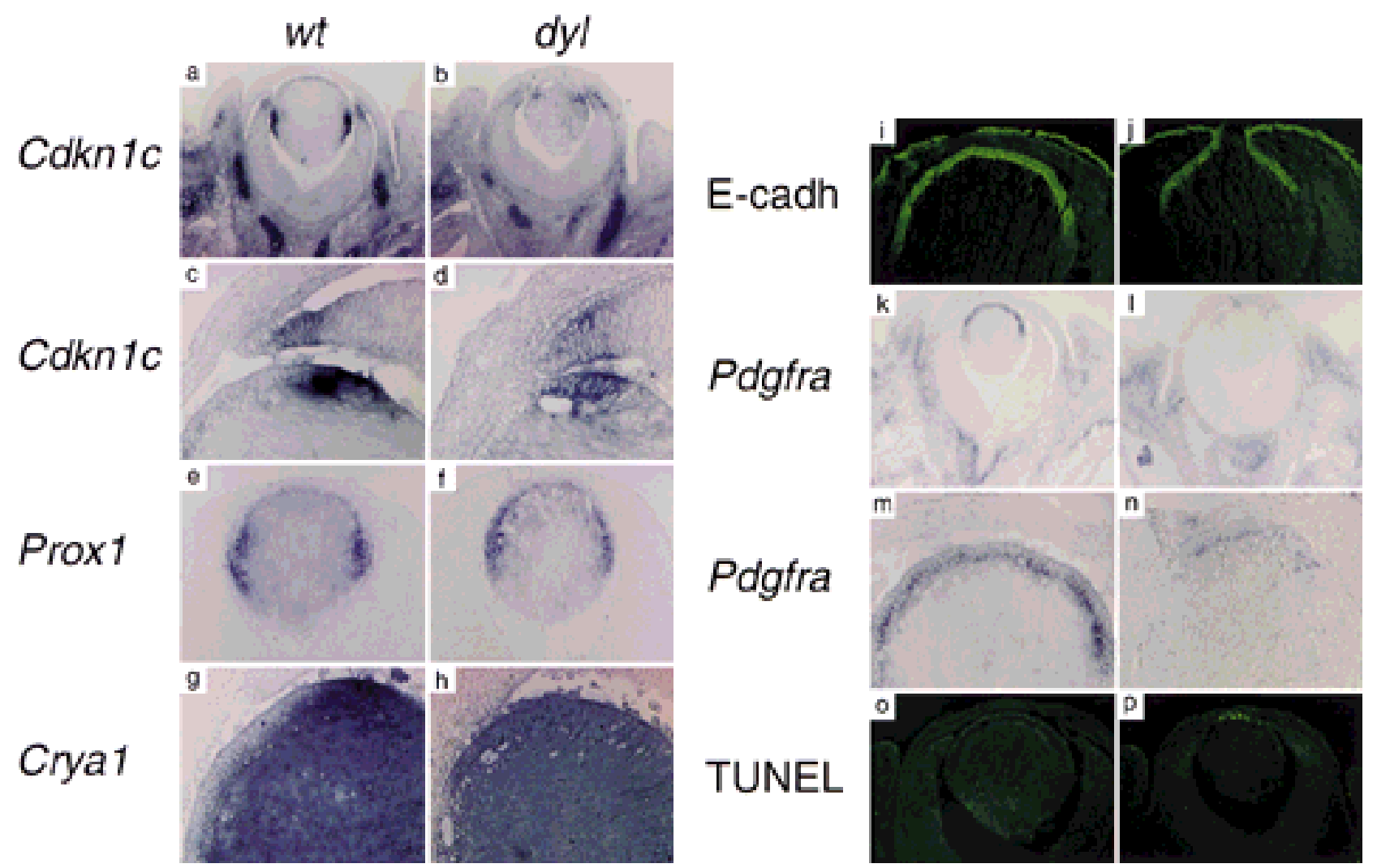

Figure 6. The lens epithelial cells in $d y l$ mutants are eliminated by premature differentiation and apoptosis. In situ hybridization $(a, b, g, h, k, l, m, n)$, immunohistochemistry $(i, j)$, and TUNEL assay $(o, p)$. The $C d k$ inhibitor $C d k n 1 c$ is expressed in the equatorial cells of the lens $(a, c)$. In the $d y l$ mutant the expression level of $C d k n 1 c$ is reduced and the expression is displaced anteriorly, overlapping with that of FoxE3 $(b, d)$. Prox1 is mainly expressed in the equatorial, nonepithelial cells $(e)$, but in the $d y l$ mutant the highest expression level is seen in the posterior part of the epithelium $(f)$. Cryal expression is low in the epithelium and highest in the equatorial zone $(g)$. In $d y l$, Crya1 is evenly expressed throughout the lens, except in the most anterior cells $(h)$. E-cadherin is expressed in the epithelium of wild-type (i) and dyl (j) lens reaching further to the posterior in wild-type. Pdgfra is expressed in the lens epithelium $(k, m)$, but is down-regulated and restricted to the most anterior cells in $d y l(1, n)$. The anterior cells in the lens epithelium undergo apoptosis in $d y l$ mutants $(p)$, whereas no signs of cell death can be detected in wild-type lens $(o)$. All sections are from E14.5 embryos.

main group have nonaromatic amino acids in either of the two positions corresponding to Phe-93 and Phe-98. These proteins invariably deviate from consensus in several other positions of the aromatic rosette and thus appear to rely on different intramolecular interactions. Deletion of, or mutations in, the human forkhead gene FOXC1 (previously known as FREAC3 or FKHL7) cause malformations in the anterior segment of the eye (Mears et al. 1998; Nishimura et al. 1998). In one extended pedigree the FOXC1 mutation that cosegregates with the eye defects is a single nucleotide transition that substitutes a serine for a phenylalanine in the forkhead domain (Phe112-Ser) (Nishimura et al. 1998). Phe-112 of FOXC1 corresponds to one of the residues in FoxE3 (Phe-98) that is mutated in $d y l$ mice and in both cases is the phenylalanine replaced by a serine. The symptoms of patients carrying the Phe-112-Ser mutation in FOXC1 are as severe as in families where the gene is deleted, which indicates that Phe-112-Ser is a null allele. By analogy, we predict that Phe-98-Ser is a null allele of FoxE3. In conclusion, each of the two substitutions in the forkhead domain of
FoxE3 found in $d y 1$ mice would be predicted to obliterate DNA binding. The presence of two such mutations, together with the colocalization of $d y l$ and FoxE3 in the mouse genome, cosegregation of the mutations with the $d y l$ phenotype and defects in $d y l$ mice being confined to the tissue where FoxE3 is expressed, strongly suggests that mutations in FoxE3 are responsible for the $d y l$ phenotype.

During normal development, the lens vesicle closes and separates from the ectoderm, a process that fails in dyl mice (Fig. 3; Sanyal and Hawkins 1979) and implies FoxE3 as an essential regulator of this event. In later stages of lens development, FoxE3 appears to be necessary for the growth and survival of the lens epithelium. In the absence of functional FoxE3 (i.e., in $d y 1$ mutants), initial anteroposterior polarization of the lens vesicle proceeds normally; by E13.5-14.5 a defined anterior epithelium is morphologically distinguishable and can also be identified by the presence of FoxE3 mRNA and Ecadherin. This polarity is thought to depend on differences in growth factor content between the aqueous and 
vitreous humor that bathe the anterior and posterior surfaces of the lens, respectively (Hyatt and Beebe 1993; Schulz et al. 1993; Klok et al. 1998; Potts et al. 1998). Lens fiber differentiation also appears to be independent of FoxE3, as judged by the expression of crystallins (Brahma and Sanyal 1984, 1987), and primary fibers fill the lumen of lens vesicles in wild-type and mutant alike. However, although the cells of a normal lens epithelium enter a phase of intense cell division, which provides the progenitors of the secondary lens fibers, the epithelial cells of a dyl mutant fail to proliferate and eventually disappear. One reason for this appears to be a changed expression pattern of genes known to promote cell cycle arrest. The posterior part of the lens epithelium, the germinative zone, is normally the area in which the most rapid growth occurs, but in the absence of FoxE3 activity, Cdkn1c and Prox1 are expressed here. Cdkn1c blocks cell cycle progression directly by inhibiting Cdks and Proxl indirectly by activating $C d k n 1 c$. The cells in what should have been the germinative zone respond to lack of FoxE3 activity by growth arrest, as illustrated by loss of expression of Pcna and Mki67 and lack of BrdU incorporation. Judged by the elevated level of Crya1 expression and the reduced range of E-cadherin expression, the fate of the most posterior of the epithelial cells instead appears to be premature differentiation.

Cells in the equatorial zone of the lens, immediately adjacent to the epithelium, show a dramatic reduction in $C d k n 1 c$ expression in the $d y 1$ mutant. The link between FoxE3 activity in the epithelial cells and Cdkn1c expression in the neighboring cells can only be speculated on, but may involve a paracrine factor produced by the epithelium, the synthesis of which depends on FoxE3.

Although the posterior part of the lens epithelium in dyl mutants show signs of cell cycle arrest and premature differentiation, the most anterior cells express Ecadherin, but not $C d k n 1 c$ or high levels of Crya1. However, the lack of BrdU incorporation show that, in spite of this, no proliferation occurs. Instead, these cells appear to be eliminated by programmed cell death. Pdgfra, encoding the PDGF $\alpha$ receptor, is highly expressed in the lens epithelium and this expression appears to depend in part on FoxE3, as seen by the diminished expression level in $d y l$ lens. The ligand, PDGF A, is secreted into the aqueous humor by the ciliary body, the iris, and the corneal endothelium and stimulates growth of the lens epithelium (Brewitt and Clark 1988; Potts et al. 1994; Reneker and Overbeek 1996). That Pdgfra is important for the response of the epithelium is shown by the developmental lens defects with reduced number of secondary lens fibers seen in Patch/Patch mouse embryos, homozygous for a null mutation in Pdgfra (MorrisonGraham et al. 1992). Therefore, it seems likely that the reduced Pdgfra expression contributes to the failure of $d y 1$ lens epithelium to proliferate, but the more severe phenotype of $d y l$ compared to Patch mutants suggests that other types of growth factor signaling may also depend on FoxE3. The aqueous humor contains survival factors for the epithelial cells of the lens (Hyatt and Beebe 1993; Ishizaki et al. 1993; Renaud et al. 1994;
Chow et al. 1995) and the apoptotic fate of these cells in the $d y 1$ mutant implies FoxE3 as a component in the generation or reception of such signals.

Classic transplantation experiments in chick embryos demonstrated the importance of the lens epithelium for cornea development (Coulombre and Coulombre 1964; Genis-Galvez 1966; Genis-Galvez et al. 1967) and recent work on mouse embryos with null mutations in the forkhead gene FoxC1 (Mf1) shows that formation of the anterior chamber depends on proper differentiation of the posterior corneal endothelium (Kidson et al. 1999). The cornea in $d y l$ mutants appears poorly differentiated and lacks the dense, highly structured appearance of a normal cornea (Fig. 3c-f). The anterior chamber is missing (Fig. 3c,d) and the posterior surface of the cornea adheres to the lens, presumably due to a poorly developed corneal endothelium (Fig. 3e,f). The nature of the signal produced by the lens epithelium that induces differentiation of the cornea remains unknown, but it is likely to depend, at least partially, on FoxE3, either directly or indirectly through maintenance of the lens epithelium.

The eye defects of $d y l$ mice resemble clinical findings in certain cases of anterior segment dysgenesis, in particular Peters' anomaly (Peters 1906; Stone et al. 1976), which is characterized by keratolenticular adhesion, central leucoma, microphthalmia, and adhesion of the iris. Occasionally, Peters' anomaly is caused by mutations in PAX6 (Hanson et al. 1994), but in the majority of cases this gene can be excluded and the cause of the defects remains unknown (Churchill et al. 1998). The human homolog of FoxE3, FOXE3, located at 1p32 (Larsson et al. 1995), must be considered a candidate gene for this disorder.

\section{Materials and methods}

Cloning of FoxE3

A genomic $129 / \mathrm{Sv} \lambda$ library (Stratagene) was screened with a probe from the human FOXE3 gene (Larsson et al. 1995) and overlapping FoxE3 clones were isolated. A 7-kb BglII fragment centered around the FoxE3 forkhead box was subcloned and sequenced on a Beckman CEQ2000 using GPS-1 transposon insertions (New England Biolabs).

\section{PCR amplification and sequencing}

DNA was prepared from Balb/c (Charles River) and $d y l / d y l$ mice (Jackson Laboratory, Maine) and the FoxE3 gene was amplified in two overlapping pieces with the primers (GGGATGGGGCCCAGAGACTGACTC and CGCAGGAAGCTACCGTTGTCGAAC; GCCCTACTCATACATCGCGCTCAT and TGGAGGAGGGCAGGGAAGGCTTAG). PCR products from $d y l$ and wild-type mice were sequenced directly with the same primers used for amplification.

Genotyping of FoxE3 ${ }^{\mathrm{T} 277 \mathrm{C}}$

A 270-nucleotide fragment from the forkhead box of FoxE3 was amplified from tail biopsies with the primers GCCCTACTCATACATCGCGCTCAT and CGCAGGAAGCTACCGTTGTCGAAC. Digestion of the product with MnlI generated three fragments from the wild-type allele $(150,114$, and 6 nucleotides) 
and four fragments from the FoxE3 $3^{T 277 C}$ allele $(114,82,68$, and 6 nucleotides), which were separated on $3 \%$ MetaPhor agarose gels.

\section{Chromosome localization}

Fluorescence in situ hybridization of mouse metaphase chromosomes (Helou et al. 1998) was performed with a 9-kb digoxigenin-labeled probe containing FoxE3. Hybridization signals were visualized with FITC-anti digoxigenin and banding patterns by DAPI counterstaining.

\section{Histological sections}

Paraffin sections $(3 \mu \mathrm{m})$ of heads from newborn (3 days) wildtype or $d y l$ mice and cryostat sections $(8 \mu \mathrm{m})$ from embryos were stained with eosin and hematoxylin.

\section{In situ hybridization and immunohistochemistry}

In situ hybridizations of whole mount mouse embryos (Rosen and Beddington 1994) and cryosections (Henrique et al. 1995) were performed with digoxigenin-labeled antisense RNA probes. The FoxE3 probe consists of a 1-kb fragment from the 3' end of the gene. Plasmids used to generate probes for Pdgfra were kindly provided by Dr. C. Betsholtz (Göteborg University, Sweden), for Mki67 by Dr. H. Igarashi (Kumamoto University, Japan), and for Crya1 by Dr. J. Piatigorsky (National Eye Institute, Bethesda, Maryland). Probes for Pcna were generated from IMAGE cDNA clone No. 605791. Unique parts of $C d k n 1 c$ (pos 200-931 in the sequence with GenBank accession no. U22399) and Prox1 (pos 132-869 in the sequence with GenBank accession no. AF061576) were amplified by PCR from genomic mouse DNA and riboprobes were transcribed from promoters appended to the primers. The authenticity of PCR products and cDNA clones were verified by DNA sequencing. No signals were observed when control sense probes were used. Radioactive in situ hybridizations of cryosections were performed as described (Mahlapuu et al. 1998) with two ${ }^{33}$ P-labeled antisense oligonucleotides from FoxE3 (CAGGGAAGGCTTAGCCCAAGCAAGGCTCGGGGACCCAGCGAATTG and CTCAGGCTGCAAGCCCAACAGGCTGTCCAGGCGGAAGAG). E-cadherin was detected with a rat monoclonal antibody (kindly provided by Dr. H. Semb) and visualized with a FITC-conjugated secondary antibody (DAKO).

\section{BrdU and TUNEL}

Kits from Roche Biochemicals and fluorescence microscopy were used to identify BrdU incorporation and apoptotic cells by the TUNEL assay in cryosections. In vivo BrdU labeling of embryos was achieved by intraperitoneal injection of $0.1 \mu$ mole of $\mathrm{BrdU} / \mathrm{g}$ body weight, $4 \mathrm{hr}$ before the pregnant females were sacrificed.

\section{Acknowledgments}

This work was supported by the Swedish Cancer Foundation (grant no. 3517-B98-05XAC), Magnus Bergvall's Foundation, Assar Gabrielsson's Foundation, and The Medical Research Fund of Tampere University Hospital. The authors are grateful to Khalil Helou and Göran Levan for help with FISH and permission to use their fluorescence microscope; to Christer Betsholtz, Joram Piatigorsky, and Hideya Igarashi for plasmid clones; to Henrik Semb (Göteborg University, Sweden) for the E-cadherin antibody; to Malin Hulander, Linda Karlsson, and Stefan Norlin for advice on in situ hybridizations; to Mattias Ormestad, Mariavittoria Sparacio, and Ulla Jukarainen for technical assistance and to an anonymous reviewer for valuable suggestions.

The publication costs of this article were defrayed in part by payment of page charges. This article must therefore be hereby marked "advertisement" in accordance with 18 USC section 1734 solely to indicate this fact.

\section{Note}

The GenBank accession no. for the FoxE3 locus $(7 \mathrm{~kb})$ is AF142647.

\section{References}

Brahma, S.K. and S. Sanyal. 1984. Immunohistochemical studies of lens crystallins in the dysgenetic lens (dyl) mutant mice. Exp. Eye Res. 38: 305-311.

1987. Ontogeny of alpha-crystallin polypeptides during the lens development of a mutant mouse. Curr. Eye Res. 6: 1291-1297.

Brewitt, B. and J.I. Clark. 1988. Growth and transparency in the lens, an epithelial tissue, stimulated by pulses of PDGF. Science 242: 777-779.

Chow, R.L., G.D. Roux, M. Roghani, M.A. Palmer, D.B. Rifkin, D.A. Moscatelli, and R.A. Lang. 1995. FGF suppresses apoptosis and induces differentiation of fibre cells in the mouse lens. Development 121: 4383-4393.

Churchill, A.J., A.P. Booth, R. Anwar, and A.F. Markham. 1998. PAX 6 is normal in most cases of Peters' anomaly. Eye 12: 299-303.

Clark, K.L., E.D. Halay, E. Lai, and S.K. Burley. 1993. Co-crystal structure of the HNF-3/fork head DNA-recognition motif resembles histone H5. Nature 364: 412-420.

Coulombre, A.J. and J.L. Coulombre. 1964. Lens development. The role of the lens in eye growth. J. Exp. Zool. 156: 39-48.

Fujiwara, M., T. Uchida, N. Osumi-Yamashita, and K. Eto. 1994. Uchida rat (rSey): A new mutant rat with craniofacial abnormalities resembling those of the mouse Sey mutant. Differentiation 57: 31-38.

Furuta, Y. and B.L.M. Hogan. 1998. BMP4 is essential for lens induction in the mouse embryo. Genes \& Dev. 12: 37643775 .

Gehring, W.J. 1996. The master control gene for morphogenesis and evolution of the eye. Genes Cells 1: 11-15.

Genis-Galvez, J.M. 1966. Role of the lens in the morphogenesis of the iris and cornea. Nature 210: 209-210.

Genis-Galvez, J.M., L. Santos-Gutierrez, and A. Rios-Gonzalez. 1967. Causal factors in corneal development: an experimental analysis in the chick embryo. Exp. Eye Res. 6: 48-56.

Glaser, T., L. Jepeal, J.G. Edwards, S.R. Young, J. Favor, and R.L. Maas. 1994. PAX6 gene dosage effect in a family with congenital cataracts, aniridia, anophthalmia and central nervous system defects. Nature Genet. 7: 463-471.

Hanson, I.M., J.M. Fletcher, T. Jordan, A. Brown, D. Taylor, R.J. Adams, H.H. Punnett, and V. van Heyningen. 1994. Mutations at the PAX6 locus are found in heterogeneous anterior segment malformations including Peters' anomaly. Nature Genet. 6: 168-173.

Helou, K., L. Walter, E. Günther, and G. Levan. 1998. Cytogenetic orientation of the rat major histocompatibility complex (MHC) on chromosome 20. Immunogenetics 47: 166169.

Henrique, D., J. Adam, A. Myat, A. Chitnis, J. Lewis, and D. Ish-Horowicz. 1995. Expression of a Delta homologue in prospective neurons in the chick [see comments]. Nature 375: 787-790.

Hyatt, G.A. and D.C. Beebe. 1993. Regulation of lens cell growth and polarity by an embryo-specific growth factor and by inhibitors of lens cell proliferation and differentiation. Development 117: 701-709. 
Ishizaki, Y., J.T. Voyvodic, J.F. Burne, and M.C. Raff. 1993. Control of lens epithelial cell survival. J. Cell Biol. 121: 899-908.

Jordan, T., I. Hanson, D. Zaletayev, S. Hodgson, J. Prosser, A. Seawright, N. Hastie, and V. van Heyningen. 1992. The human PAX6 gene is mutated in two patients with aniridia. Nature Genet. 1: 328-332.

Jänne, P.A., J.M. Rochelle, P.A. Martin-DeLeon, D. Stambolian, M.F. Seldin, and R.L. Nussbaum. 1995. Mapping of the 75$\mathrm{kDa}$ inositol polyphosphate-5-phosphatase (Inpp5b) to distal mouse chromosome 4 and its exclusion as a candidate gene for dysgenetic lens. Genomics 28: 280-285.

Kamachi, Y., M. Uchikawa, J. Collignon, R. Lovell-Badge, and H. Kondoh. 1998. Involvement of Sox1, 2 and 3 in the early and subsequent molecular events of lens induction. Development 125: 2521-2532.

Kaufmann, E. and W. Knöchel. 1996. Five years on the wings of fork head. Mech. Dev. 57: 3-20.

Kidson, S.H., T. Kume, K. Deng, V. Winfrey, and B.L. Hogan. 1999. The forkhead/winged-helix gene, Mf1, is necessary for the normal development of the cornea and formation of the anterior chamber in the mouse eye. Dev. Biol. 211: 306-322.

Klok, E.j., N.H. Lubsen, C.G. Chamberlain, and J.W. McAvoy. 1998. Induction and maintenance of differentiation of rat lens epithelium by FGF-2, insulin and IGF-1. Exp. Eye Res. 67: 425-431.

Larsson, C., M. Hellqvist, S. Pierrou, I. White, S. Enerbäck, and P. Carlsson. 1995. Chromosomal localization of six human forkhead genes, FREAC-1 (FKHL5), -3 (FKHL7), -4 (FKHL8), -5 (FKHL9), -6 (FKHL10), and -8 (FKHL12). Genomics 30: 464-469.

Loosli, F., S. Winkler, and J. Wittbrodt. 1999. Six3 overexpression initiates the formation of ectopic retina. Genes \& Dev. 13: 649-654.

Mahlapuu, M., M. Pelto-Huikko, M. Aitola, S. Enerbäck, and P. Carlsson. 1998. FREAC-1 contains a cell type-specific transcriptional activation domain and is expressed in epithelialmesenchymal interfaces. Dev. Biol. 202: 183-195.

Mathers, P.H., A. Grinberg, K.A. Mahon, and M. Jamrich. 1997. The Rx homeobox gene is essential for vertebrate eye development. Nature 387: 603-607.

McAvoy, J.W. 1980. Induction of the eye lens. Differentiation 17: 137-149.

Mears, A.J., T. Jordan, F. Mirzayans, S. Dubois, T. Kume, M. Parlee, R. Ritch, B. Koop, W. Kuo, C. Collins, J. Marshall, D.B. Gould, W. Pearce, P. Carlsson, S. Enerbäck, J. Morisette, S. Battacharaya, B. Hogan, V. Raymond, and M.A. Walter. 1998. Mutations of the Forkhead/Winged-helix gene, FREAC3/FKHL7, in patients with axenfeld-rieger anomaly. Am. J. Hum. Genet. 63: 1316-1328.

Morgenbesser, S.D., B.O. Williams, T. Jacks, and R.A. DePinho. 1994. p53-dependent apoptosis produced by $\mathrm{Rb}$-deficiency in the developing mouse lens. Nature 371: 72-74.

Morrison-Graham, K., G.C. Schatteman, T. Bork, D.F. BowenPope, and J.A. Weston. 1992. A PDGF receptor mutation in the mouse (Patch) perturbs the development of a non-neuronal subset of neural crest-derived cells. Development 115: $133-142$.

Nishiguchi, S., H. Wood, H. Kondoh, R. Lovell-Badge, and V. Episkopou. 1998. Sox1 directly regulates the gamma-crystallin genes and is essential for lens development in mice. Genes \& Dev. 12: 776-781.

Nishimura, D.Y., R.E. Swiderski, W.L.M. Alward, C.C. Searby, S.R. Patil, S.R. Bennet, A.B. Kanis, J.M. Gastier, E.M. Stone, and V.C. Sheffield. 1998. The forkhead transcription factor gene FKHL7 is responsible for glaucoma phenotypes which map to 6p25. Nature Genet. 19: 140-147.
Ogino, H. and K. Yasuda. 1998. Induction of lens differentiation by activation of a bZIP transcription factor, L-Maf. Science 280: $115-118$.

Oliver, G., A. Mailhos, R. Wehr, N.G. Copeland, N.A. Jenkins, and P. Gruss. 1995. Six3, a murine homologue of the sine oculis gene, demarcates the most anterior border of the developing neural plate and is expressed during eye development. Development 121: 4045-4055.

Orr-Urtreger, A. and P. Lonai. 1992. Platelet-derived growth factor-A and its receptor are expressed in separate, but adjacent cell layers of the mouse embryo. Development 115: 10451058.

Peters, A. 1906. Über angeboren Defektbildung der Descementschen Membran. Klin. Mbl. Augenheilk. 44: 27-40.

Piatigorsky, J. 1981. Lens differentiation in vertebrates. A review of cellular and molecular features. Differentiation 19: $134-153$.

Pierrou, S., M. Hellqvist, L. Samuelsson, S. Enerbäck, and P. Carlsson. 1994. Cloning and characterization of seven human forkhead proteins: Binding site specificity and DNA bending. EMBO J. 13: 5002-5012.

Porter, F.D., J. Drago, Y. Xu, S.S. Cheema, C. Wassif, S.P. Huang, E. Lee, A. Grinberg, J.S. Massalas, D. Bodine, F. Alt, and H. Westphal. 1997. Lhx2, a LIM homeobox gene, is required for eye, forebrain, and definitive erythrocyte development. Development 124: 2935-2944.

Potts, J.D., S. Bassnett, S. Kornacker, and D.C. Beebe. 1994. Expression of platelet-derived growth factor receptors in the developing chicken lens. Invest. Ophthalmol. Vis. Sci. 35: 3413-3421.

Potts, J.D., S. Kornacker, and D.C. Beebe. 1998. Activation of the Jak-STAT-signaling pathway in embryonic lens cells. Dev. Biol. 204: 277-292.

Renaud, F., L. Oliver, S. Desset, J. Tassin, N. Romquin, Y. Courtois, and M. Laurent. 1994. Up-regulation of aFGF expression in quiescent cells is related to cell survival. J. Cell. Physiol. 158: 435-443.

Reneker, L.W. and P.A. Overbeek. 1996. Lens-specific expression of PDGF-A alters lens growth and development. Dev. Biol. 180: 554-565.

Rosen, B. and R. Beddington. 1994. Detection of mRNA in whole mounts of mouse embryos using digoxigenin riboprobes. Methods Mol. Biol. 28: 201-208.

Sanyal, S. and R.K. Hawkins. 1979. Dysgenetic lens (dyl)-A new gene in the mouse. Invest. Ophthalmol. Vis. Sci. 18: $642-645$.

Sanyal, S., R. Van Nie, J. De Moes, and R.K. Hawkins. 1986. Map position of dysgenetic lens (dyl) locus on chromosome 4 in the mouse. Genet. Res. 48: 199-200.

Schulz, M.W., C.G. Chamberlain, R.U. de Iongh, and J.W. McAvoy. 1993. Acidic and basic FGF in ocular media and lens: Implications for lens polarity and growth patterns. Development 118: 117-126.

Spemann, H. 1901. Über korrelation in der entwicklung des auges. Verh. Anat. Ges. 15: 61-79.

Stone, D.L., K.R. Kenyon, W.R. Green, and S.J. Ryan. 1976. Congenital central corneal leukoma (Peters' anomaly). Am. J. Ophthalmol. 81: 173-193.

Wigle, J.T., K. Chowdhury, P. Gruss, and G. Oliver. 1999. Prox1 function is crucial for mouse lens-fibre elongation. Nature Genet. 21: 318-322.

Zhang, P., C. Wong, R.A. DePinho, J.W. Harper, and S.J. Elledge. 1998. Cooperation between the Cdk inhibitors p27(KIP1) and $\mathrm{p} 57(\mathrm{KIP} 2)$ in the control of tissue growth and development. Genes \& Dev. 12: 3162-3167. 


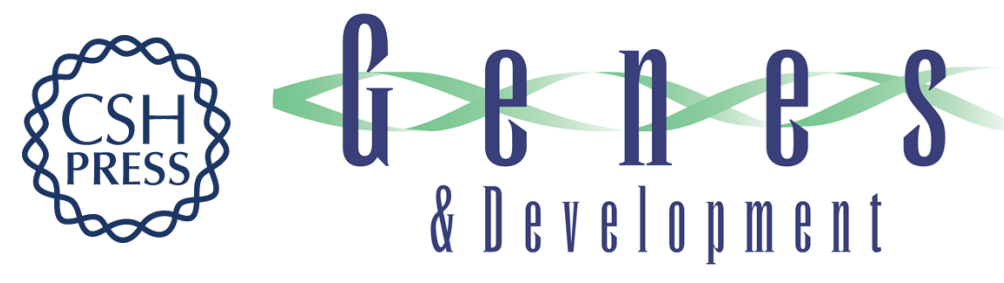

\section{A forkhead gene, FoxE3, is essential for lens epithelial proliferation and closure of the lens vesicle}

Åsa Blixt, Margit Mahlapuu, Marjo Aitola, et al.

Genes Dev. 2000, 14:

Access the most recent version at doi:10.1101/gad.14.2.245

References This article cites 52 articles, 17 of which can be accessed free at: http://genesdev.cshlp.org/content/14/2/245.full.htmI\#ref-list-1

License

Email Alerting

Receive free email alerts when new articles cite this article - sign up in the box at the top Service right corner of the article or click here.

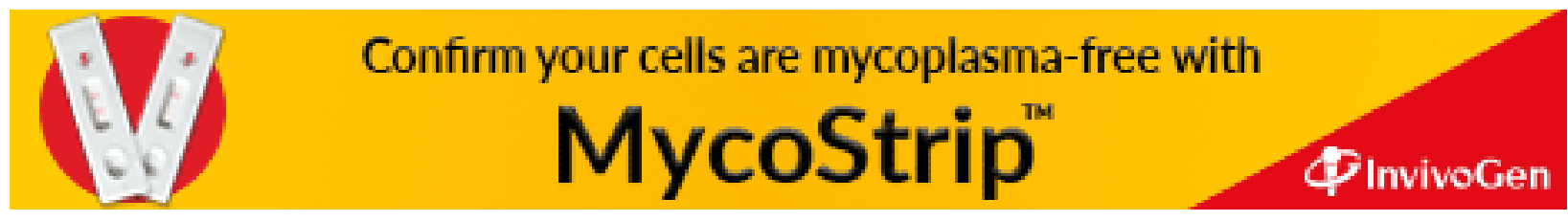

